\title{
Espacio público efectivo en Manizales y Medellín, Colombia: evaluación cuantitativa de su generación y distribución en dos momentos normativos
}

\author{
Espaço público efetivo em Manizales e Medellín, Colômbia: \\ avaliação quantitativa de sua geração e distribuição em \\ dois momentos normativos
}

Effective public space in Manizales and Medellín, Colombia: quantitative evaluation of its generation and distribution in two normative moments

\section{Maria Fernanda Cardenas [a] (DD), Tania Giraldo-Ospina [b] [D]}

\section{[a] Universidad Nacional de Colombia, Medellín, Antioquia, Colombia}

[b] Universidad Nacional de Colombia, Manizales, Caldas, Colombia

Cómo citar: Cardenas, M. F., \& Giraldo-Ospina, T. (2021). Espacio público efectivo en Manizales y Medellín, Colombia: evaluación cuantitativa de su generación y distribución en dos momentos normativos. urbe. Revista Brasileira de Gestão Urbana, v.13, e20210075. https://doi.org/10.1590/2175-3369.013.e20210075

\section{Resumen}

El espacio público -EP- urbano es fundamental para las ciudades sostenibles, resilientes, habitables. Su relevancia es reconocida por políticos, instituciones y academia. No obstante, su materialización no es la proyectada, más si se trata de ciudades andinas en relieves montañosos como Manizales y Medellín en Colombia. Este trabajo exploró las directrices sobre EP en las normas nacionales y locales establecidas en los planes de ordenamiento territorial -POT- de los municipios, de los cuales hay dos versiones, dos momentos. Así mismo, evaluó los EP al inicio de cada POT para determinar cuánto nuevo EP se logró generar y en qué partes de la ciudad, como un indicio para analizar la equidad en su distribución. Se encontraron cambios importantes entre las dos generaciones de POT en ambas ciudades en cuanto a los discursos, y concepciones del EP, intenciones declaradas de equidad, concentración de EP en algunas zonas urbanas y carencia en otras. Las ciudades siguen lejos de lograr las metas planteadas en los indicadores cuantitativos y, además, están perdiendo EP por ocupación de privados. Los retos son cada vez mayores, pero además de lo cuantitativo, es necesario considerar características de calidad, accesibilidad y conectividad que dan vitalidad a estos espacios.

Palabras clave: Espacio público efectivo. Zonas verdes urbanas. Planificación urbana. Políticas públicas.

\section{Resumo}

O espaço público -EP- urbano é essencial para cidades sustentáveis, resilientes e habitáveis. Sua relevância é reconhecida por políticos, instituições e acadêmicos. Porém, sua concretização não ocorre conforme o planejado, 
principalmente se se tratar de cidades andinas em relevos montanhosos como Manizales e Medellín na Colômbia. Este trabalho explorou as diretrizes sobre PE nas regulamentações nacionais e locais estabelecidas nos planos de ordenamento territorial -POT- dos municípios, dos quais existem duas versões, dois momentos. Da mesma forma, avaliou o EP no início de cada POT para determinar quanto EP novo foi gerado e em que pontos da cidade, como um indicativo para analisar a equidade na sua distribuição. Mudanças importantes foram encontradas entre as duas gerações de POT nas duas cidades em termos de discursos, e nas concepções de EP, intenções declaradas de equidade, concentração de EP em algumas áreas urbanas e carência em outras. As cidades ainda estão longe de atingir as metas estabelecidas nos indicadores quantitativos e, além disso, estão perdendo EP devido à ocupação privada. Os desafios são crescentes, mas além dos quantitativos, é preciso considerar características de qualidade, acessibilidade e conectividade que dão vitalidade a esses espaços.

Palavras-chave: Espaço público eficaz. Zonas verdes urbanas. Planejamento urbano. Políticas públicas.

\section{Abstract}

The urban public space -PS- is essential for sustainable, resilient, livable cities. Its relevance is recognized by politicians, institutions, and academia. However, its materialization is not as planned, especially in Andean cities in mountainous reliefs such as Manizales and Medellín in Colombia. This work explored the directives on PS in the national and local regulations established in the territorial ordering plans -POT- of the municipalities, of which there are two versions, two moments. Likewise, it evaluated the PS at the beginning of each POT to determine how much new PS was generated and in which parts of the city, as an indication to analyze the equity in its distribution. Important changes were found between the two generations of POT in both cities in terms of discourses, and in the conceptions of PS, declared intentions of equity, concentration of PS in some urban areas and lack in others. Cities are still far from achieving the goals set in the quantitative indicators and, furthermore, they are losing PS due to private occupation. The challenges are increasing, but in addition to the quantitative, it is necessary to consider characteristics of quality, accessibility and connectivity that give vitality to these spaces.

Keywords: Effective public space. Urban green areas. Urban planning. Public politics.

\section{Introducción}

Las ciudades son el hábitat del ser humano moderno, cuyos problemas y retos toman cada vez mayores dimensiones. Estas deben repensar su desarrollo buscando espacios urbanos -EP- resilientes, sostenibles y equitativos (ONU, 2016). Los ciudadanos tienen el derecho a gozar de una estructura urbana equilibrada, solidaria y de carácter arquitectónico (Talavera-Dávila, 2017), lo cual se concreta a través de los EP, donde las relaciones entre los habitantes, el poder y la ciudadanía se materializan y se expresan (Borja y Muxí, 2000). El EP además es un medio para asegurar la equidad territorial, en tanto permite a los ciudadanos realizarse social y ambientalmente, siempre y cuando este sea efectivo, es decir, que se pueda utilizar permanentemente, a cualquier hora del día y por diferentes motivos (Gehl, 2014).

El EP materializado con zonas verdes cobra relevancia por sus connotaciones paisajísticas (Caquimbo, 2008; López et al., 2018; Mesa Carranza y López Bernal, 2016), por los beneficios para la salud física y mental de los ciudadanos (Lee y Maheswaran, 2011), por las mejoras ambientales (Gómez, 2005), la conectividad entre los bosques urbanos y el territorio circundante (PNNC, 2015), porque aportan resiliencia climática (Grafakos et al., 2019) y resiliencia sísmica al ser potenciales sitios de refugio temporal, de respuesta y recuperación en caso de eventos catastróficos, cuando son EP conectados y accesibles (Allan y Bryant, 2014; Koren y Rus, 2019; French et al., 2019).

Adicionalmente, los EP de calidad traen ventajas políticas para las administraciones municipales porque mejoran la reputación y el marketing de las ciudades (Carmona et al., 2008); incrementan la conciencia ambiental, la participación ciudadana, mejoran la calidad de vida de los habitantes y ayudan a alcanzar las metas globales en relación con la sostenibilidad urbana, calidad del aire, prevención de desastres naturales y mitigación del cambio climático (Alcaldía de Medellín, 2017). No obstante, el enfoque 
del EP como un componente de la competitividad urbana, también puede poner en peligro la protección del medio ambiente y la oferta de EP para todos (Madanipour et al., 2014).

El interés por el EP es creciente, dado su valor económico, social y ambiental; sin embargo, se encuentra una dicotomía en su producción y gestión: por una parte, se generan espacios que a causa de la sub-gestión se vuelven lugares descuidados, invadidos y excluyentes; mientras que otros EP son sobre-gestionados, resultando en áreas privatizadas y orientadas al consumo (Pojani y Maci, 2015; Carmona 2010a, 2010b). Así, la calidad del espacio público es producto de la gestión, la cual puede incorporar procesos de cogestión y autogestión con participación de la comunidad y el sector privado (Jansson et al., 2020).

Aunque en las ciudades latinoamericanas, donde la desigualdad en los ingresos de la población es muy marcada, no se ha evaluado suficientemente la evolución y distribución de los EP (Macedo y Haddad, 2016); las disparidades en el acceso a estos espacios han sido demostradas en relación con los ingresos socioeconómicos y la etnia (Rigolon et al., 2018). En el caso de las ciudades colombianas, los EP son insuficientes (PNNC, 2015); y la generación de los nuevos no alcanza a suplir las demandas crecientes ocasionadas por el incremento en la población urbana.

Los beneficios generados por los EP deben llegar a todos los ciudadanos, para lo cual es relevante que éstos se distribuyan por toda el área urbana. Más aún, la equidad territorial indica que su distribución debería privilegiar a los ciudadanos con menores recursos, ya que ellos tienen menos posibilidades de proveerse de espacios de recreación, zonas verdes y sitios de encuentro de manera privada (Boone et al., 2009; Cárdenas et al., 2020; Ojeda Casares et al., 2008; Zuindeau, 2007).

Este trabajo analiza la producción de EP en dos ciudades de Colombia, a la vez que reflexiona sobre los marcos normativos generales y las apuestas locales y en los EP construidos en los últimos años, que permiten entender la distancia entre las directrices y políticas formuladas, y los resultados en la ciudad construida. A partir de los hallazgos en ambas ciudades, se espera identificar algunas de las causas estructurales y las posibles soluciones, a fin de armonizar lo planteado en los instrumentos de planeación con las acciones en el territorio. Las ciudades elegidas para el ejercicio son Manizales y Medellín, dos ciudades andinas con limitaciones para su expansión dada su topografía: la primera situada en la cima de una montaña, la segunda asentada en el fondo de un valle estrecho. Los espacios públicos de ambas ciudades se registraron por escalas territoriales (urbana, comunas y barrios), contrastando los cambios entre los dos Planes de Ordenamiento Territorial -POT-, que representan dos momentos de planificación de las ciudades.

\section{Metodología}

\section{Sitios de estudio}

Este trabajo analiza la distribución y producción de EP en Manizales y Medellín, más que con el ánimo de hacer análisis comparativos entre ellas, se consideran casos de estudio de ciudades andinas con limitaciones geográficas para su expansión urbana. No obstante, el estudio de ambas ciudades permite hacer interpretaciones y sugerencias generales para superar la distancia entre las políticas y la materialización del espacio público. Las dos ciudades se sitúan sobre la Cordillera Central (Figura 1). 

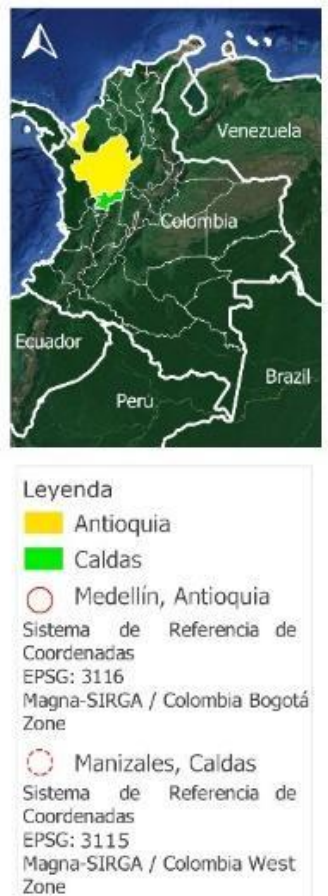

Zone
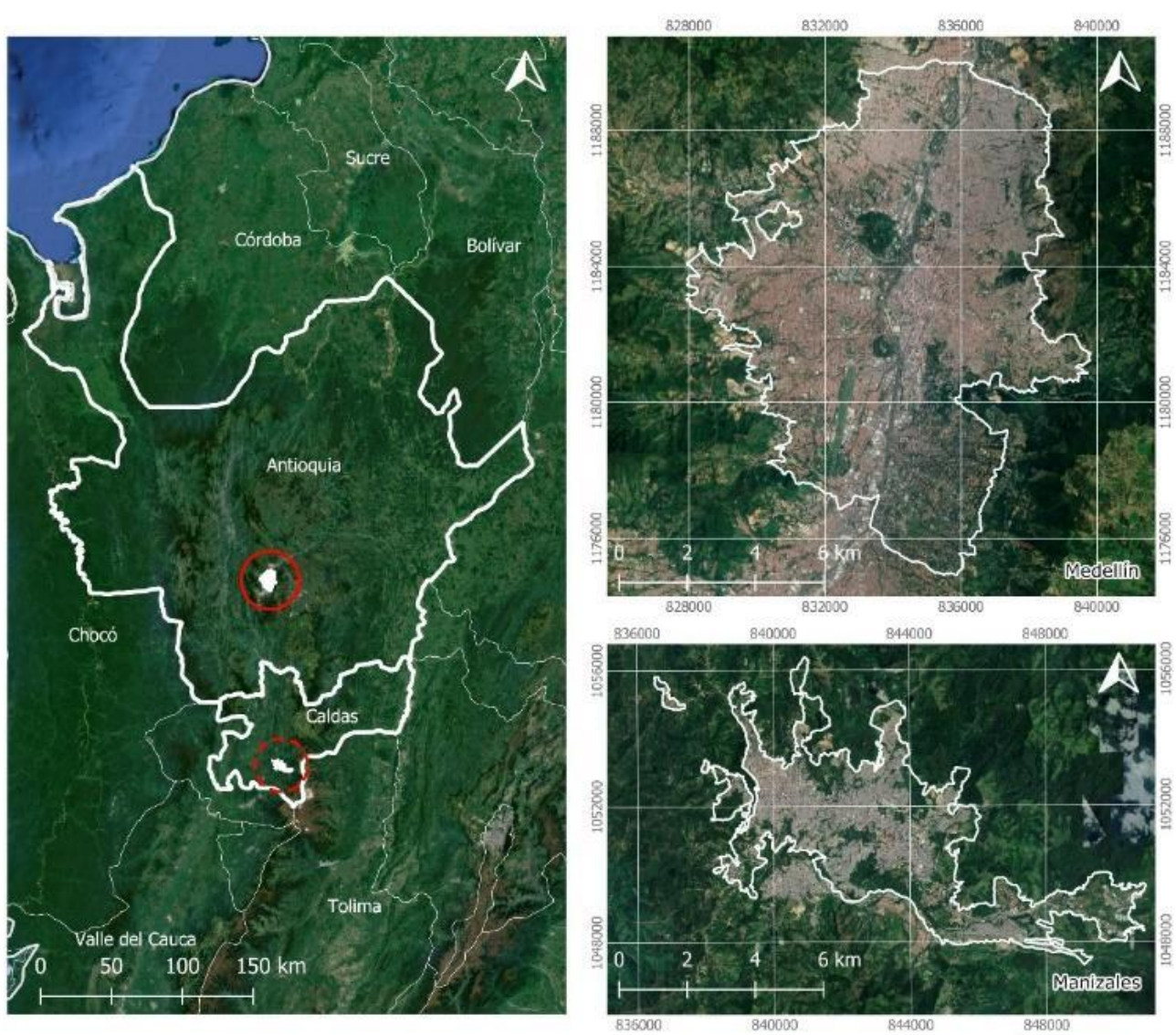

Figura 1 - Localización de Manizales, Caldas y de Medellín, Antioquia, en Colombia. Fuente: Medellín (2014); Manizales (2017).

Manizales es la capital del departamento de Caldas, se ubica en el centro-occidente de Colombia, sobre la vertiente occidental de la Cordillera Central, a 2150 metros de altitud, sobre un área de $38,15 \mathrm{~km}^{2}$, que albergan 375.432 habitantes (DANE, 2018). Su topografía montañosa, las fuertes pendientes de las laderas y la presencia de áreas de interés ambiental en el suelo urbano, han restringido la expansión de la ciudad, generando un tejido urbano discontinuo. La ciudad está distribuida político-administrativamente en 11 comunas y 114 barrios.

Medellín es la capital del departamento de Antioquia. Se asienta en la parte más ancha del valle de Aburrá, a $1475 \mathrm{msnm}$. Tiene una extensión de $110,2 \mathrm{~km}^{2}$ de suelo urbano y $270 \mathrm{~km}^{2}$ de suelo rural. La zona urbana está distribuida político-administrativamente en 16 comunas y 249 barrios (Medellín, 2014). La población se estima en 2.371.330 habitantes (DANE, 2018).

\section{Normativa municipal relacionada con espacio público}

El territorio es la expresión espacial de las políticas ambientales, sociales, económicas y culturales de una sociedad (Gómez Orea y Gómez Villarino, 2013), por tanto, se busca analizar los resultados en las áreas nuevas de EP en relación con las políticas que orientan el ordenamiento territorial en las dos ciudades. Para ello se revisaron los POT de primera y de segunda generación, con énfasis en los EPE. Estos instrumentos de planificación orientan el modelo de ocupación y son de obligatorio cumplimiento en los municipios colombianos según la Ley 388 de 1997 (Colombia, 1997).

\section{Caracterización del espacio público}

La identificación de los EPE en las ciudades estudiadas se hizo con base en la cartografía oficial de los POT municipales. La información se analizó para dos momentos de planificación, contrastando el EP en 
términos cuantitativos. Los dos momentos son los años de adopción de los POT, que para Manizales fueron 2001 y 2017, y para Medellín 2006 y 2014. En Medellín se tomó 2018 como un tercer momento, debido a la actualización de información hecha para ese año.

A partir de la cartografía se calcularon las áreas totales de EPE para cada área urbana, por comunas y barrios, identificando dónde y cuánto EPE nuevo se generó durante los períodos evaluados. Mediante recorridos virtuales y en campo, se actualizó el inventario de EPE y se identificaron las áreas que se encuentran ocupadas por privados, perdiendo su connotación de EPE y los beneficios que generan.

\section{Resultados}

\section{El espacio público en la normatividad municipal}

La Constitución Política de Colombia (1991) abarca disposiciones y deberes en cuanto al EPE, buscando la protección de su integridad y la del medio ambiente en general, regula la relación de la sociedad con la naturaleza: establece que el Estado debe garantizar el derecho a gozar de un ambiente sano ; al igual que la protección de la integridad del EPE y su destinación al uso común, prevaleciendo sobre el interés particular; señala que los habitantes también deben velar por la conservación de su entorno; determina que el Estado debe racionalizar la economía para mejorar la calidad de vida de los habitantes, la distribución equitativa de las oportunidades y beneficios del desarrollo y un medio ambiente sano adecuado,. Este marco constitucional obligó a las administraciones municipales a planificar y a definir regulaciones específicas para el EPE (Ceballos, 2008), que competen a las instituciones públicas, pero también a los particulares.

El espacio público efectivo -EPE- constituye una parte de todo el EP definido en la Ley 9 de 1989 (Colombia, 1989). Este concepto se introdujo con el Decreto 1504 de 1998 (PRC, 1998) que indica que el EPE está conformado por parques, plazas, plazoletas y zonas verdes, y hace referencia al EP de carácter permanente. En este trabajo se asume que el EP reconocido en los instrumentos de planificación territorial municipal se refieren al EPE definido por este Decreto. En la categoría de parques se incluyen los parques infantiles, deportivos y ornamentales y, aunque los andenes son los conectores por excelencia entre los EP, no hacen parte de los EPE. Otro conjunto de EP no efectivos, según la normatividad colombiana, son los antejardines, retiros viales y cesiones urbanísticas. Ambas ciudades entienden el indicador de EPE de la misma manera, porque atienden el requerimiento establecido en el Decreto, aunque las áreas a incorporar en la categoría de EPE quedan a criterio de la administración municipal, lo que posibilita la inclusión de espacios como separadores ambientales, glorietas vehiculares y laderas, que no cumplen con la condición de ser áreas de encuentro, desvirtuando el propósito de estos lugares.

El indicador de EPE en Colombia está definido en el mismo Decreto, en unidad de área por habitante. Es un indicador cuantitativo que no considera la calidad, la distribución espacial en las ciudades, el clima ni las densidades urbanas, condiciones que varían al interior de las ciudades y entre ellas. Así mismo, el Decreto estableció una meta de $15 \mathrm{~m}^{2}$ de EPE por habitante para las ciudades, replicando un estándar internacional atribuido a la Organización Mundial de la Salud, que sugiere entre 10 y $15 \mathrm{~m}^{2}$ de zonas verdes por habitante (DNP, 2012). La Ley 2037 de 2020 (Colombia, 2020) exige a los municipios disponer de un inventario de EPE actualizado, para calcular indicadores cuantitativos y cualitativos.

\section{El espacio público en la normatividad de Manizales}

El municipio adoptó su primer POT mediante el Acuerdo Municipal 508 de 2001 (Manizales, 2001), al cual se hicieron revisiones en 2003 (Manizales, 2003) y 2007 (Manizales, 2007). El POT del 2001 establece la visión del municipio a 25 años, donde promueve a Manizales como "un eje del conocimiento que cuente con un desarrollo tecnológico, industrial y agroindustrial que se caracterice por su conectividad física a través de la intervención del espacio público", en particular EPE que se caracterizará por involucrar el 
entorno natural para hacer uso del paisaje como parte integral del desarrollo cotidiano de sus habitantes, brindando mecanismos para su apropiación.

El POT de 2001 hace referencia a la falta de EPE de calidad en la ciudad, hecho que se ha visto en aumento debido al crecimiento urbano. También plantea la inclusión de EPE en zonas de ladera, aunque estos requieren de diseños innovadores que satisfagan criterios de accesibilidad, seguridad y uso, necesarios para su disfrute, considerando que algunos EPE poseen o requieren tratamientos geotécnicos (Giraldo-Ospina y Vásquez-Varela, 2021) y articulación con la ciudad. Este POT resalta el carácter integrador y articulador del EPE, y la necesidad de concebirlo como elemento integral, lo cual es relevante en una ciudad como Manizales por sus condiciones geográficas que propician dominio visual sobre el paisaje, dentro del cual se destacan las rondas hídricas del río Chinchiná, la quebrada Olivares y otras corrientes que pueden ser usados como conectores del sistema natural.

El POT de 2001 también menciona la carencia de instrumentos para fomentar la creación, conservación y mantenimiento de los espacios colectivos. Con el fin de solucionar el déficit cuantitativo y cualitativo, el POT plantea incorporar el paisaje natural al tejido urbano, incluyendo nuevas áreas para incrementar el índice de EPE y, a su vez, reducir la segregación e inequidad espacial.

En 2017 Manizales modificó el POT (Manizales, 2017). Planteó mejorar y crear nuevos EPE interconectados para contribuir con el mejoramiento de la habitabilidad de la ciudad. Propuso articular el EPE con la estructura ecológica, los sistemas de movilidad y los equipamientos colectivos, promoviendo un desarrollo en armonía con el paisaje natural, para lo cual se requiere determinar cuáles áreas naturales son adaptables para uso recreativo, activo y pasivo.

La propuesta del POT es suplir el déficit de EPE con infraestructuras que posibiliten diversas actividades económicas, la convivencia social, la preservación de los atributos geográficos del lugar y la conservación del tejido urbano. Para alcanzar estos objetivos se planteó la adecuación de escombreras municipales clausuradas como EPE, construcción de parques lineales en las rondas hídricas, incorporación de laderas urbanas, suelos de protección y de interés ambiental como parte constitutiva del EPE, e implementar la elaboración de Planes Especiales de Actuación -PEA-, con el fin de enmarcar las actuaciones y proyectos del sistema, considerando las nuevas demandas de la población y con esquemas de competencias que permitan una gestión adecuada.

Según el POT (Manizales, 2017), la ciudad contaba a 2015 con $6,77 \mathrm{~m}^{2} /$ hab de EPE, considerando 368.655 habitantes. Para el año 2027 propone una meta de 22,05 $\mathrm{m}^{2} / \mathrm{hab}$, con proyección de 397.816 habitantes urbanos.

\section{El espacio público en la normatividad de Medellín}

El municipio adoptó su primer POT mediante el Acuerdo Municipal 62 de 1999 (Medellín, 1999). Este documento reconoce una relación directa entre la calidad del EP con la calidad de la ciudad, y determina que el EPE es el "estructurante principal de la construcción de ciudad y de ciudadanía", al tiempo que lo señala como uno de los elementos orientadores del modelo de ocupación del territorio. No obstante, este Acuerdo no plantea proyectos con metas específicas sobre EPE, más allá de la generación o recuperación de algunos miradores.

Las ideas mencionadas sobre EP se retoman casi textualmente en el POT ajustado (Acuerdo Municipal 46 de 2006). Entre los lineamientos del nuevo POT, se destaca la "prioridad al espacio público; a su generación, consolidación, mejoramiento y apropiación social, (...) donde tenga prioridad el peatón, y el transporte público tenga prevalencia sobre el particular".

Los elementos constitutivos del EPE se dividen en naturales, conformados por el sistema hidrográfico, el orográfico y los ecosistemas estratégicos; y artificiales, retomando las definiciones del decreto 1504. Para este último grupo, se proponen estrategias como la construcción de nuevo EPE en centralidades que carezcan de él, a través del pago de obligaciones urbanísticas, proyectos mixtos público-privados y concesiones del municipio al privado, y menciona proyectos de construcción de nuevos EPE de ámbito 
metropolitano como parques del río, parques de borde urbano, el parque Arví, parque de occidente y parque explora.

La única meta o indicador relacionado con EPE en el Acuerdo 46 aparece en el apartado sobre las cesiones urbanísticas, al señalar que dichas cesiones deben estar orientadas, entre otras cosas, a "aumentar el indicador de espacio público por habitante, aproximándolo al establecido por el Documento Colombia 2019, en el cual se establece para el horizonte de largo plazo un estándar de $10 \mathrm{~m}^{2}$ de EPE por habitante."

En 2014 Medellín renovó su POT, el cual se adoptó mediante el Acuerdo 48. Este POT tiene un discurso novedoso, evidenciando una evolución en el proceso de ordenamiento del territorio. El EPE, si bien sigue apareciendo como un elemento relevante en el imaginario de ciudad y en el modelo de ocupación del territorio, no aparece como el único sistema articulador urbano. Así mismo, reconoce que no existe una red de EPE que responda equitativamente a la distribución de la población en la ciudad (Mayorga, 2018).

La composición del EPE sigue siendo la misma, aunque este POT especifica cuáles áreas se incorporarán al EPE y cómo. Entre los criterios establecidos para la generación de nuevos EPE se incluye la equidad, según la cual se priorizarán aquellas zonas donde los indicadores señalan mayores déficits.

En el anexo del componente programático del POT se encuentran programas orientados a la generación de nuevas áreas para incorporar al EPE de esparcimiento y encuentro, así como al mejoramiento, restauración, mantenimiento y restitución de este tipo de espacios. En los proyectos no especifican las estrategias para desarrollarlos, pero el texto permite inferir que se espera generar la mayor parte de los EPE nuevos a partir de cesiones derivadas de las actuaciones urbanísticas, así como mediante intervenciones en proyectos a escala de ciudad que apuntan a la generación de EPE para alcanzar una meta de $7 \mathrm{~m}^{2}$ / habitante (Mora Eusse, 2019), aunque se aclara que esta meta es a largo plazo y que el área se calculará de acuerdo con la población proyectada a 2030 (Medellín, 2014).

\section{Similitudes y contrastes entre ambas ciudades}

Manizales y Medellín tienen inventarios de EPE desactualizados e imprecisos en los registros oficiales. El incremento de EPE en Manizales, se debió en parte a la actualización de la medición y a la incorporación de zonas verdes, y no a la construcción de nuevos espacios (Manizales cómo vamos 2019). Con la actualización cartográfica de este trabajo se hallaron 4,88 ha ocupadas por privados a 2021 en Manizales, de los cuales el $45 \%$ corresponde a la comuna Ciudadela del Norte y el $33 \%$ a la comuna Tesorito, donde predominan usos residenciales y comerciales. El área ocupada representa el 0,0003\% del EPE de la ciudad. En Medellín, la verificación de la ocupación de los EPE del POT señala que 78,08 ha, casi el 10\% del EPE total, están invadidas con construcciones, cercas, parqueaderos, etc. Estas situaciones se encontraron especialmente en los sectores altos en la ladera, en las comunas 7-Robledo (24\%), 8-Villa Hermosa (20\%), 16-Belén (8\%) y en la 9-Buenos Aires (7\%). Estos hallazgos evidencian la falta de apropiación social y cultural, y la ausencia de una gestión adecuada, en los términos de la Política Nacional de Espacio Público (DNP, 2012).

Ambas ciudades cuentan con instrumentos de planeación y gestión que permiten tomar medidas para incrementar sus EPE en cantidad y calidad, aunque algunos no se han reglamentado. En Manizales, aunque el POT de 2017 no definió instrumentos verdes, incluyó instrumentos financieros, económicos y de pago por bienes y servicios ambientales con participación del sector privado para el manejo y sostenibilidad de las áreas protegidas, porque mediante ingresos corrientes del municipio serían insuficientes. Los beneficios del incremento de EPE durante estos años, también se han visto reflejados en la encuesta de percepción de la calidad de vida (Manizales cómo vamos, 2019), el nivel de satisfacción con el EPE de la ciudad casi se ha duplicado: 33\% (2012) a 63\% (2018). En Medellín, la inversión realizada proviene de recursos propios del municipio, y en lo que respecta a EPE se distribuye entre construcción y mantenimiento. En 2014 y 2015 hubo un incremento en la inversión en construcción, asociada a las etapas 1 y 2 del proyecto Parques del Río; que entre 2012-2015 ocupó 47,2\% del presupuesto anual en equipamientos (Medellín cómo vamos 2016). Durante los últimos 12 años el indicador de EP en Medellín ha fluctuado alrededor de $3,7 \mathrm{~m}^{2} / \mathrm{hab}$ 
(Medellín cómo vamos, 2019), lo que indica que los esfuerzos hechos para generar nuevo espacio público solo han logrado mantener la proporción con relación al crecimiento poblacional, pero deben generarse este tipo de espacios a mayores tasas para alcanzar la meta propuesta de $7 \mathrm{~m}^{2} / \mathrm{hab}$.

\section{Espacio público efectivo urbano desde la adopción de los POT de primera y segunda generación}

\section{Manizales: período 2001-2017}

El EPE de Manizales según el POT (Manizales, 2017) incluye los ecoparques, bulevares y separadores ambientales, aunque estas no pertenecen a las categorías definidas en el Decreto 1504 de 1998 (PRC, 1998). A pesar de incorporar estas categorías aún está lejos de alcanzar la meta de los $15 \mathrm{~m}^{2} / \mathrm{hab}$.

En el año 2001 Manizales contaba con 124,1 ha de EPE. Durante el período 2001 - 2017 se adicionaron 162,7 ha para un total de 286,8 ha. Del área nueva de espacio público generado en el período, casi 60\% (95,3 ha) está en la comuna 6 Cerro de Oro, y dentro de ella, 90,6 ha equivalentes al 95\% del nuevo EPE de la comuna, están en el barrio Viveros; evidenciando la concentración espacial del EPE generado (figura 2, figura 3a). El EPE que se entregó en la ciudad entre el primer y el segundo POT, se distribuyó en 91 de los 114 barrios. Además del barrio Viveros, los barrios con mayor incremento de espacio público fueron Sancancio (6,65 ha), La Enea (47,3 ha), San Sebastián, San Cayetano, Ciudadela La Linda y Las Colinas, con más de 32 ha de nuevo EPE cada uno (figura 3b). De los 46 barrios que a 2001 carecían de EPE, 16 aún no tienen (figura 3c), y con la actualización del inventario realizado en este trabajo se halló que el barrio Residencias Manizales perdió el poco EPE de zona verde que tenía para ser utilizado como acceso a estacionamientos. Por otro lado, en 30 barrios que no tenían, ahora cuentan con EPE, que suma 106,3 ha; o 15,7 ha sin incluir el ecoparque Los Yarumos del barrio Viveros. En total, entre 2001-2017 Manizales incrementó su EPE en 131,2\%.
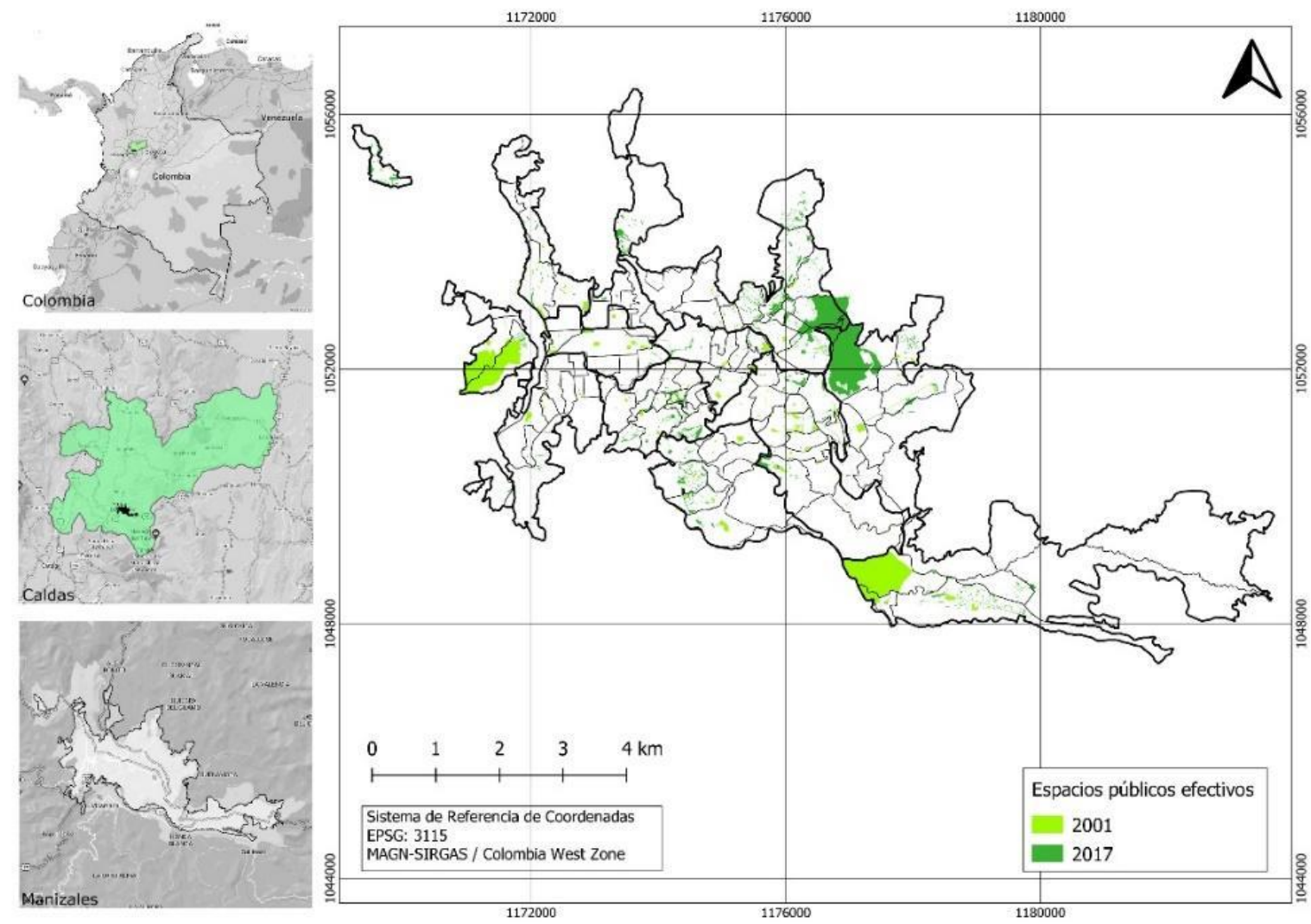

Figura 2 - EPE de Manizales en los años 2001 y 2017. Fuente: Elaboración propia a partir de Alcaldía de Manizales (2021) e imagen de satélite @2020 de Google. 


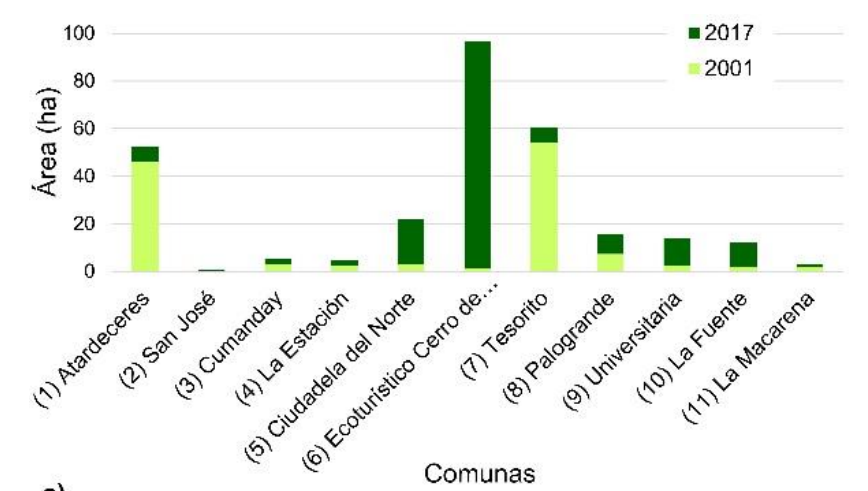

a)

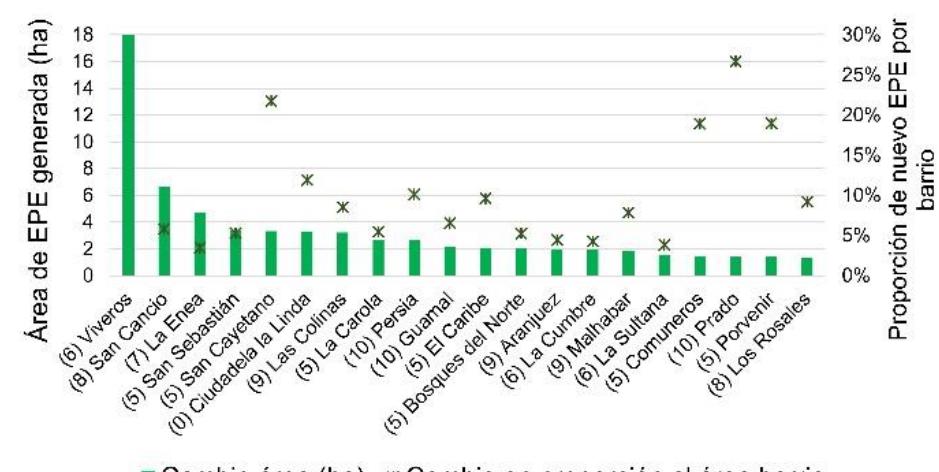

b)

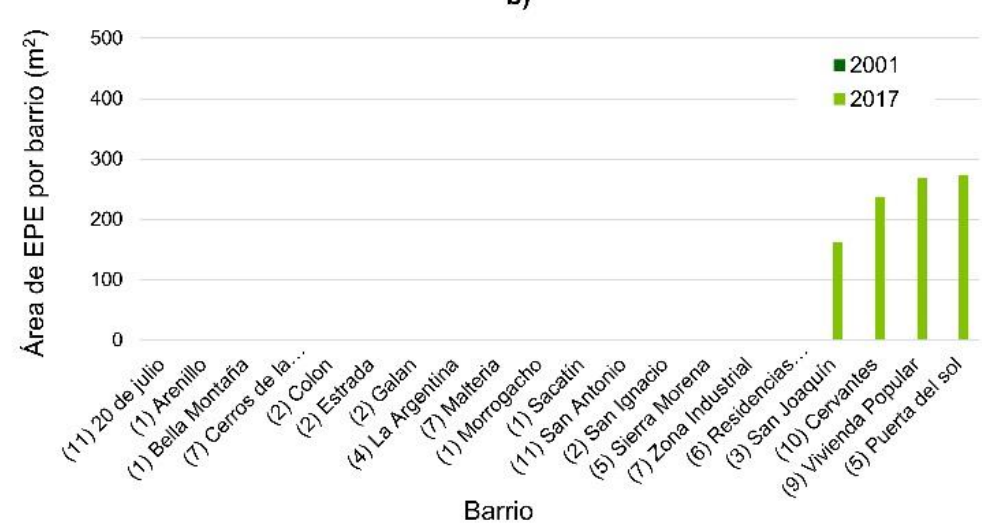

Figura 3 - Cambios en el EPE de Manizales entre 2001-2017. a) cambios por comunas; b) los 20 barrios con mayores incrementos (en área y en proporción) de EPE; c) los 20 barrios con menos EPE en 2001 y escenario en 2017. Los números entre paréntesis señalan la comuna a la que pertenece el barrio. En b no aparece el área total de nuevo EPE del barrio Viveros ya que invisibilizaba los valores de los demás barrios. Fuente: Elaboración propia.

\section{Medellín: periodos 2006-2014-2018}

Las intenciones de incrementar el EPE en la ciudad se concretaron mediante intervenciones de pequeña escala entre 2006-2014, cuando se adicionaron 26,6 ha de nuevo EPE a las 774 ha que existían. El aumento fue de 3,4\% y se repartió en 54 barrios. Los mayores incrementos se dieron en Carlos E. Restrepo (6,4 ha, comuna 11), en Villatina (2,5 ha, comuna 8), en la Nueva Villa del Aburrá (2,5 ha, comuna 16) y en Moravia (1,7 ha, comuna 4). Durante el período 2014-2018 se desarrollaron nuevos EPE a escala de ciudad, como la etapa $1 \mathrm{~A}$ de parques del río, una gran intervención que permitió recuperar la conectividad de la ciudad con el río Medellín. Asimismo, se entregaron 12 Unidades de Vida Articulada -UVA-, EPE generados en predios urbanos donde las empresas públicas de Medellín -EPM- tienen tanques para la distribución de agua. Con este tipo de intervenciones, Medellín sumó 37 ha de nuevos EPE, distribuidos por toda la ciudad (figura 4), un 4,6\% adicional con respecto al EPE que había en 2014.

A escala de comuna, las que más EPE ganaron entre 2006-2018 fueron la 11-Laureles-Estadio (6,6 ha), la 8-Villa Hermosa (3,9 ha) y la 3-Manrique (3,8 ha). Entre 2014-2018 las comunas que más ganaron EPE fueron la 14-Poblado (9,7 ha), la 1-Popular (4,8 ha) y la 3-Manrique (4 ha) (figura 5a); lo cual demuestra que las comunas con las mejores condiciones socioeconómicas (11 y 14) concentran gran parte de las inversiones en EPE en la ciudad, aunque se nota un esfuerzo por distribuirlos entre los distintos sectores de Medellín: la figura 5b señala los 20 barrios donde hubo mayores cambios en el periodo 2006-2018, distribuidos en 12 de las 16 comunas. La figura 5 c indica que se ha tratado de subsanar el déficit en algunos de los barrios con menos EPE, no obstante, según los datos de 2018 aún hay 8 barrios que carecen de este. 


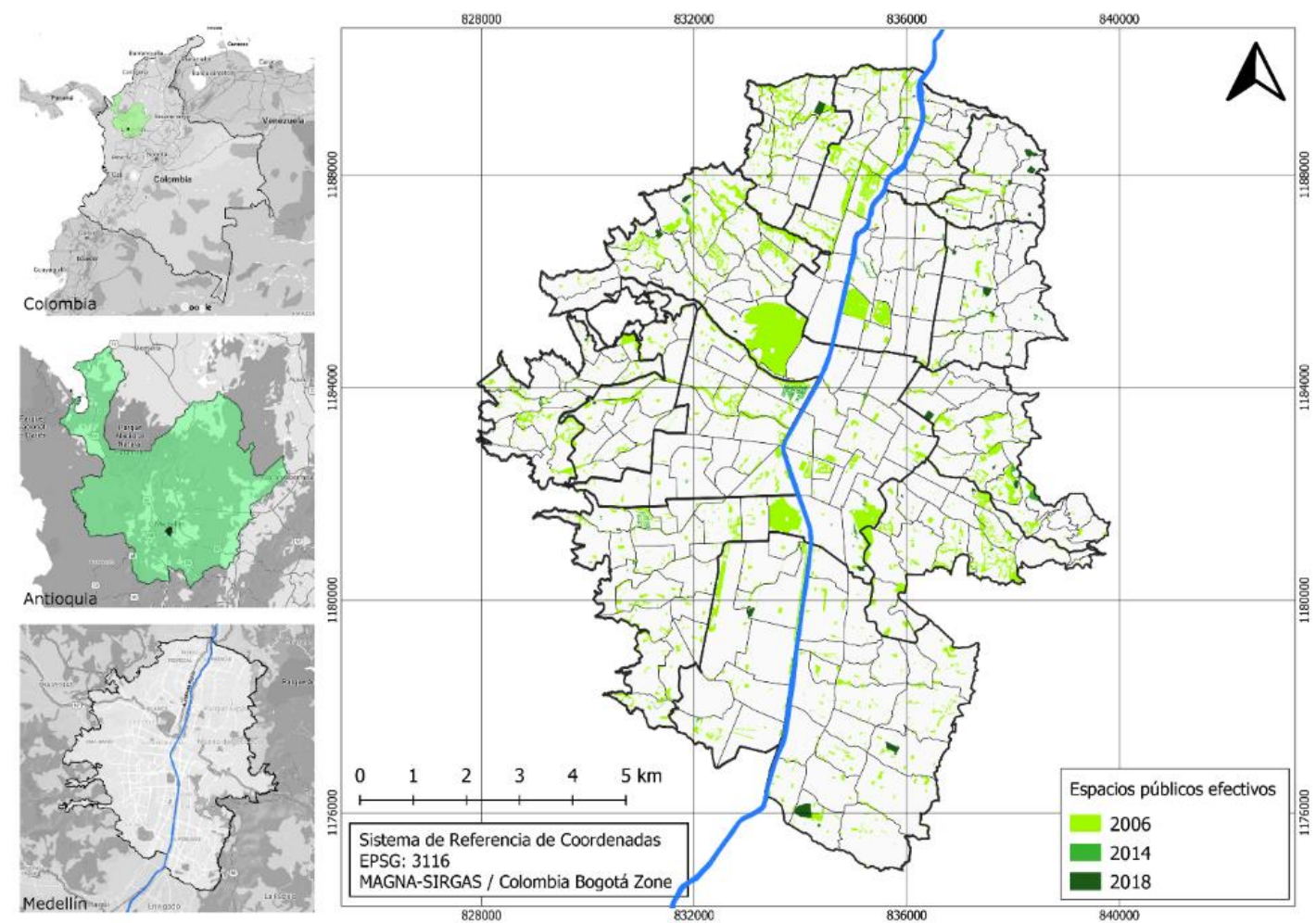

Figura 4 - EPE de Medellín en los años 2006, 2014 y 2018. Fuente: Elaboración propia a partir de Medellín (2006 y 2014), actualización a 2018 e imagen de satélite @2020 de Google.
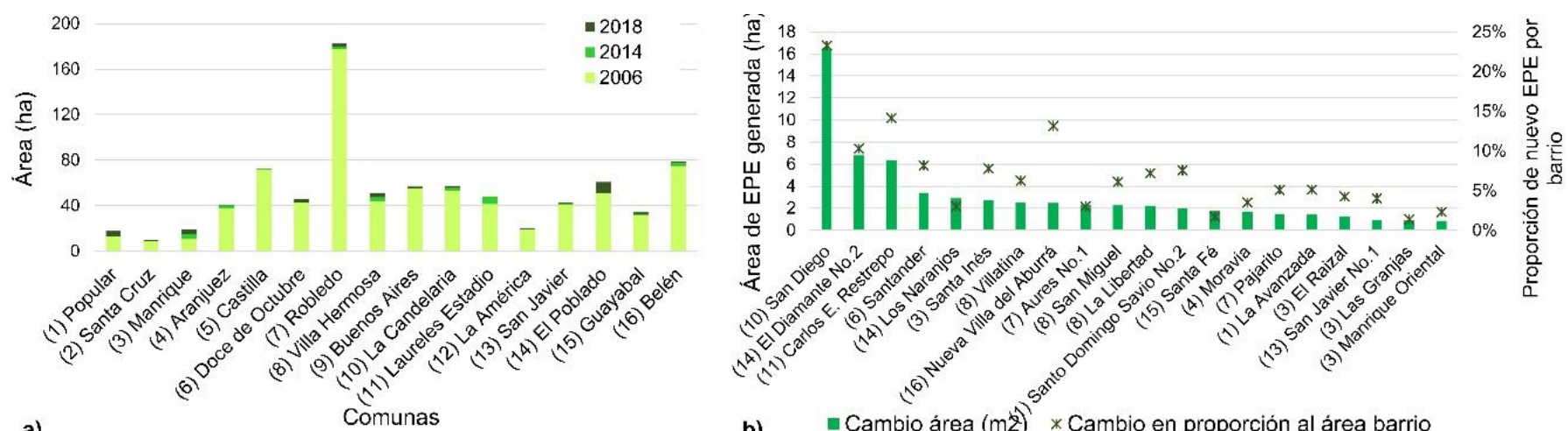

a)

$$
\text { Comunas }
$$

c)

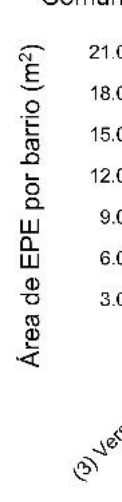

21.000

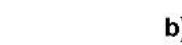

b) Cambio área $(\mathrm{m} 2)^{\prime} *$ Cambio en proporción al área barrio
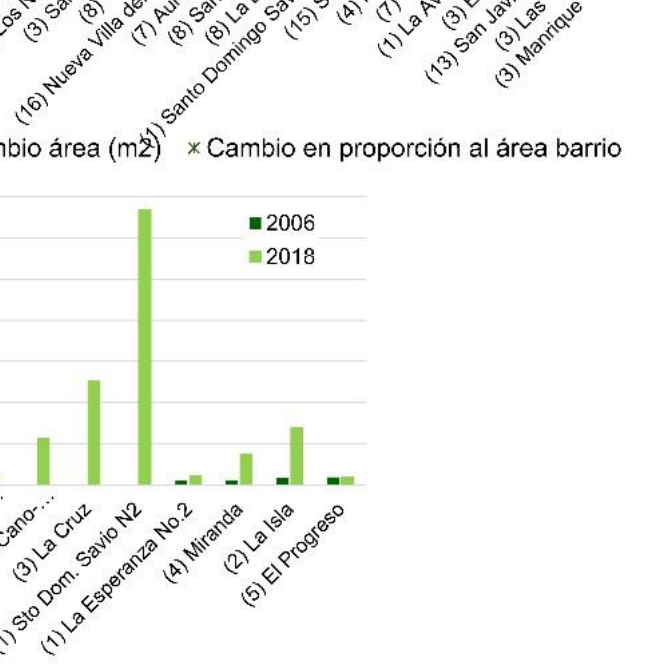

Figura 5 - Cambios en el EPE de Medellín entre 2006-2018. a) cambios por comunas; b) los 20 barrios con mayores incrementos (en área y en proporción) de EPE; c) los 20 barrios con menos EPE en 2006 y área de EPE a 2018. Entre paréntesis se indica el número de la comuna a la que pertenece el barrio. Fuente: Elaboración propia. 


\section{Discusión}

El mejoramiento de las condiciones ambientales urbanas, especialmente en contextos topográficos complejos y limitantes como los de las ciudades de estudio, requiere del compromiso y la participación del sector público, privado y la comunidad, y de un fuerte liderazgo político; ya que los proyectos se materializan en hechos transformadores y las acciones son efectivas cuando se realiza una gestión ambiental colectiva (Posada, 2014). Más aún cuando el EPE y las zonas verdes urbanas cumplen funciones sociales, ambientales, culturales y económicas, que son reconocidas en las políticas públicas, plasmadas en los POT de estas ciudades. No obstante, la provisión de nuevos EPE se dificulta cuando el relieve restringe la expansión urbana, las áreas aptas para urbanizar ya están ocupadas, y los procesos de densificación urbana no producen EP acordes al incremento poblacional. Medellín recurrió a la reutilización de los tanques de distribución de agua de EPM para generar espacios para las comunidades, denominados UVA, una estrategia para aprovechar espacios con potencial, apelando a la multifuncionalidad. Por otro lado, Parques del Río ha requerido una gran inversión para soterrar parcialmente una autopista que corre paralela al río, a fin de situar el parque donde antes transitaban los vehículos. Otra estrategia implementada en ambas ciudades ha sido generar nuevos EPE en los bordes urbanos y en las laderas no aptas para expansión urbana, con limitantes de accesibilidad y de conectividad; si bien estas nuevas áreas contribuyen a la estructura ecológica, no aportan como espacios de encuentro.

Además de buscar el cumplimiento de compromisos internacionales como la Nueva Agenda Urbana (ONU, 2017), que alude a la creación de EP seguros, incluyentes, accesibles, verdes y de calidad, o de los objetivos de desarrollo sostenible ODS como el ODS11 para ciudades y comunidades sostenibles; y de las políticas nacionales que orientan las actuaciones en materia de gestión ambiental urbana, movilidad, y EP; las administraciones municipales deben orientar sus esfuerzos a lograr las metas trazadas, a equilibrar los usos de los territorios y a mejorar las condiciones de vida de sus habitantes.

Además, los municipios enfrentan retos financieros para generar nueva oferta y mantener adecuadamente los EPE existentes. Afrontarlos requiere de instituciones locales fuertes que implementen el marco normativo de manera efectiva (Smolka, 2013; De la Sala et al., 2019; Kaw et al., 2020), y de instrumentos de gestión y financiación como monitoreo de la propiedad inmobiliaria, transferencia de derechos de construcción y desarrollo, captura del valor adicional del suelo, pago por bienes y servicios ambientales, cesiones y compensaciones urbanísticas, entre otros que permitan incrementar las áreas de EPE. Los municipios cuentan con una amplia opción de estrategias y herramientas para generar y conservar el EPE; a las cuales se suman los modelos flexibles o mixtos que posibilitan una gestión del EP a largo plazo con responsabilidades compartidas entre la comunidad y/o el sector privado, con un gobierno local que direccione las inversiones y las estrategias y regule el aprovechamiento económico. Es decir, el municipio indica dónde se construyen los EPE y sus características, define si es más conveniente construir un gran EPE de carácter municipal o varios parques barriales, y los demás actores aportan en la conservación de los lugares, el desarrollo de eventos y encuentros sociales, y la priorización de los proyectos según las necesidades de su entorno. En este sentido, se resalta que el POT de Medellín reconoce la importancia de distribuir los EPE equitativamente entre la población urbana; aunque en la práctica, viene concentrando las cesiones urbanísticas para la construcción de grandes parques en laderas poco accesibles, con altas pendientes, situadas en el borde o por fuera del perímetro urbano.

En los casos estudiados se notan prácticas débiles en cuanto a la gestión del EPE, expresadas en falta de control y carencia de inventarios actualizados y sin regular el aprovechamiento económico de los elementos constitutivos del EPE (Linares, 2018), como lo establece la Política Nacional de Espacio Público (DNP, 2012). Manizales tiene previsto en su POT vigente caracterizar y regular el uso de los EPE, lo que constituye un avance en materia de planeación urbana, pero aún no se ha reglamentado ni implementado. La evolución cuantitativa de los EPE en ambas ciudades se ha visto afectada por la gestión, que se ha enfocado en perspectivas de corto plazo, a cargo principalmente del Estado. Una gestión dinámica, continua y de largo plazo entre la comunidad y el sector privado permitiría una mayor eficacia en el uso de los 
instrumentos de planeación y gestión; con nuevos actores y prácticas se facilita el incremento de la cantidad y calidad del espacio público.

La materialización de nuevos EPE en las ciudades analizadas enfrenta varios retos: en primer lugar, la carencia de espacio urbanizable que ha obligado a las administraciones a promover densificación urbana y desarrollos en altura, lo cual conlleva a tomar decisiones sobre qué usos privilegiar. En este sentido, mientras que construir nuevos inmuebles para uso residencial o comercial implica más recaudo de impuestos, la construcción de equipamientos colectivos y EPE significan para los municipios costos de inversión y de mantenimiento a perpetuidad. Y claramente estas lógicas económicas han estado por encima de las ideas de sostenibilidad ambiental y la promesa de contribuir a la construcción de una ciudad más equilibrada, que brinde mejores condiciones y buena calidad de vida para sus habitantes. No obstante, las inversiones en EPE varían entre periodos administrativos, dejando claro que, a fin de cuentas, la aplicación de los instrumentos propuestos desde el ordenamiento territorial está sujeta a las decisiones políticas de los gobernantes de turno.

El EPE en Colombia hace referencia a un subconjunto de los EP, de los cuales las plazas, los parques deportivos o infantiles son fácilmente identificados y, generalmente, mejor mantenidos; sin embargo, la condición de público o privado de las zonas verdes no es tan clara, lo que las hacen susceptibles de ser ocupadas por particulares, o descuidadas. La diferencia en la gestión de estos EPE resulta en evoluciones diversas que, sumadas a la falta de control, vigilancia y mantenimiento, pueden incidir en la pérdida de EP, a pesar de los esfuerzos de las administraciones públicas por incrementar las áreas de EPE urbano, como lo plantea Carmona (2010a; 2010b). Adicionalmente, las problemáticas asociadas al descuido de los EPE tienen otros efectos sociales como inhibición del uso del lugar, el incremento en robos y prácticas violentas, o una imagen negativa y pérdida de confianza en las instituciones (Cárdenas-O'Byrne, 2017; Malone y Dammert, 2020), cuando se debe propender por hacer cada vez más habitables los EP con que se cuentan (Páramo y Burbano, 2012).

El interés por el EPE ha sido creciente, y es cada vez más apreciado por la ciudadanía en general, más aún cuando el mundo enfrenta retos ambientales sin precedentes, como el cambio climático, que obliga a repensar los hábitos y estilos de vida, y a valorar cada vez más los bosques urbanos y los servicios ecosistémicos que ellos prestan (Dobbs et al., 2018), buscando reconstruir ciudades más sostenibles y resilientes (Bibri y Krogstie, 2017; Borja y Muxí, 2000; Haughton, 1997; Hernández, 2009; Thompson, 2002). Además de aportar espacios abiertos y zonas verdes, el EP es clave para la transformación de los modos de transporte hacia opciones no contaminantes como las bicicletas (Sarmiento et al., 2017), aunque esta opción tiene limitantes en las ciudades analizadas en este trabajo, debido a las fuertes que presentan en algunas zonas. Adicionalmente, el contexto de pandemia por Covid-19, dejó en evidencia que gran parte de los hogares en América Latina habitan en viviendas reducidas con limitantes para realizar sus actividades cotidianas; por lo cual los EPE cobran relevancia como complemento a los espacios privados.

Sin embargo, las inequidades en la provisión de EPE halladas entre los barrios de las ciudades trascienden las disparidades en la escala urbana. Por tanto, puede decirse que persiste una brecha para reducir la segregación en la provisión de EPE, los desajustes en su distribución espacial, injusticia climática e inequidad ambiental entre los ciudadanos para disfrutar de sus beneficios.

Los hallazgos reportados en este documento concuerdan con Pérez Medina (2018), en cuanto a los avances en el interés de las administraciones públicas locales por incrementar los EPE, buscando su conexión con la naturaleza y el entorno urbano para mejorar la calidad de vida de la población y aportar a la mitigación de los efectos del cambio climático.

\section{Conclusiones}

El EPE es fundamental en las ciudades contemporáneas, cuya representación en los discursos políticos y en las normas locales de ordenamiento territorial ha venido evolucionando: en los POT de primera generación el EPE se concebía como el principal estructurante urbano y el conector físico del resto de 
elementos urbanos entre sí, y de estos con los componentes naturales del territorio, incluido el paisaje. En los POT de segunda generación, el EPE se presenta como un sistema que debe integrarse con los demás sistemas urbanos, dejando de ser el único eje ordenador y articulador. Asimismo, cada vez se reconocen más beneficios y atributos deseables al EPE y a las zonas verdes urbanas de calidad.

En ciudades como Manizales y Medellín es difícil concretar la generación de nuevos EPE y alcanzar las metas trazadas en las agendas de gobierno; no solo por la escasez de recursos debido a la falta de implementación de mecanismos de financiación del desarrollo urbano, sino por la escasez de lugares aptos para materializar nuevos EPE, y porque no han sido una prioridad para las administraciones municipales. En las nuevas normas se plantean más instrumentos y estrategias que permiten viabilizar la generación de EPE, sumadas a las consideraciones de equidad en la selección de proyectos, así como a las demandas de la población por mejores condiciones de habitabilidad, ya que el déficit de EPE es cada vez más evidente, más sentido, y la ciudadanía eventualmente exigirá una ciudad más equilibrada en la distribución de usos del suelo.

Los resultados evidencian los esfuerzos que las administraciones municipales han hecho por incrementar las áreas de EPE. En el período 2001-2017 Manizales aumentó 131\% la extensión de EPE que tenía en 2001, aunque este incremento estuvo altamente concentrado en un solo barrio, mientras que el $14 \%$ de los barrios de la ciudad aún carecen de EPE. Medellín muestra el contraste de implementar dos estrategias distintas: intervenciones de pequeña escala con las que sumó 3,4\% de EPE en 2006-2014, y proyectos a escala de ciudad que le permitieron incrementar 4,6\% en 2014-2018, cuyos beneficios se distribuyen por la ciudad con criterios de equidad. No obstante, se notan tendencias a invertir más en las comunas con mejor nivel socioeconómico, y $3 \%$ de los barrios de la ciudad aún no tienen EPE. Esto sin contar el alto porcentaje de áreas de EPE que se encontraron apropiadas por particulares.

El EPE es mucho más que áreas en un mapa de inventario o indicadores cuantitativos, es necesario también considerar el mantenimiento, fomentar la apropiación, la seguridad y brindar las condiciones para que los ciudadanos lo usen y velen por su conservación. Si bien estos gobiernos locales son conscientes de las oportunidades que ofrecen los EP, una mejor comprensión de los beneficios asociados puede facilitar la gestión para incrementar la cantidad y calidad de estos. Además de las metas trazadas en cuanto a indicadores cuantitativos de EPE por habitante, hay que considerar factores de calidad, conectividad y accesibilidad a estos espacios, de manera que contribuyan a mejorar la calidad de vida de los ciudadanos.

\section{Agradecimientos}

A la Dirección Nacional de Investigación y Laboratorios por la financiación de este trabajo a través de la Convocatoria Nacional para el Fomento de Alianzas Interdisciplinarias que articulen Investigación, Creación, Extensión e Innovación en la Universidad Nacional de Colombia 2019-2021. Código Hermes 47256. A Mara Alejandra Abril, Juan Felipe Ortega y Natalia Gaviria del semillero URBI-SIG quienes procesaron y elaboraron la cartografía.

\section{Declaración de disponibilidad de datos}

El conjunto de datos que respalda los resultados de este artículo está disponible en SciELO DATA y se puede acceder a él en https://doi.org/10.48331/scielodata.XQH495.

\section{Referencias}

Alcaldía de Medellín. (2017). Informe de calidad de vida de Medellín, 2016. Pregón SAS.

Alcaldía de Manizales (2021). «Geoportal». Gestión de Información Geográfica - Planeación Municipal. Recuperado el 25 de febrero de 2021 de http://geodata-manizales-sigalcmzl.opendata.arcgis.com. 
Allan, P., \& Bryant, M. (2014). The attributes of resilience. A tool in the evaluation and design of earthquake-prone cities. International Journal of Disaster Resilience in the Built Environment, 5(2), 109-129.

Bibri, S. E., \& Krogstie, J. (2017). Smart sustainable cities of the future: An extensive interdisciplinary literature review. Sustainable Cities and Society, 31, 183-212. https://doi.org/10.1016/J.SCS.2017.02.016

Boone, C. G., Buckley, G. L., Grove, J. M., \& Sister, C. (2009). Parks and people: An environmental justice inquiry in Baltimore, Maryland. Annals of the Association of American Geographers, 99(4), 767-787.

https://doi.org/10.1080/00045600903102949

Borja, J., \& Muxí, Z. (2000). El espacio público, ciudad y ciudadanía (2000.a ed.).

Caquimbo, S. (2008). La calidad del espacio público en la construcción del paisaje urbano. En busca de un hábitat equitativo. Revista INVI, 22(62), 75-97.

Cárdenas, M. F., Escobar, J. F., \& Gutiérrez, K. (2020). Equidad territorial en Medellín: Espacio público, amenazas naturales y calidad del aire. Estudios Socioterritoriales, 27, 1-18.

Cárdenas-O’Byrne, S. (2017). Medir el uso del espacio público urbano seguro. Sociedad y Economía. https://doi.org/10.25100/sye.v0i33.5620

Carmona, M. (2010a). Contemporary Public Space: Critique and Classification, Part One: Critique. Journal of Urban Design, 15(1), 123-148. https://doi.org/10.1080/13574800903435651

Carmona, M. (2010b). Contemporary Public Space, Part Two: Classification. Journal of Urban Design, 15(2), 157-173. https://doi.org/10.1080/13574801003638111

Carmona, M., Magalhães, C. de, \& Hammond, L. (2008). Public space: The management dimension. Routledge.

Ceballos, O. L. (2008). La cualificación de la periferia urbana y el espacio público. Una reflexión desde las políticas públicas de Bogotá. 18-19, 207-227.

Colombia. (1989, 11 de enero). Ley 9 de 1989. Por la cual se dictan normas sobre planes de desarrollo municipal, compraventa y expropiación de bienes y se dictan otras disposiciones.

Colombia. (1991, 20 de julio). Constitución Política de la República de Colombia.

Colombia. (1997, 12 de septiembre). Ley 388 de 1997. Por la cual se modifica la Ley 9a de 1989, y la Ley $3^{\text {a }}$ de 1991 y se dictan otras disposiciones.

Colombia. (2020, 27 de julio). Ley 2037 de 2020. Por la cual se modifica el artículo 6 de la Ley 388 de 1997 y se dictan otras disposiciones.

De La Sala, S., Maldonado, M., \& Alterman, R. (2019). Políticas de Suelo, Derecho Urbanístico y Cambio Climático:

Instrumentos urbanísticos-tributarios como medidas para enfrentar al Cambio Climático (Working Paper WP19SD1SP, p.

96). Lincoln Institute of Land Policy. Recuperado el 14 de junio de 2021 de

https://www.lincolninst.edu/publications/working-papers/politicas-suelo-derecho-urbanistico-cambio-climatico

Departamento Administrativo Nacional de Estadísticas - DANE. (2018). Resultados y Censo Nacional de Población y Vivienda 2018. Manizales, Caldas (p. 39). https://www.dane.gov.co/files/censo2018/informacion-tecnica/presentacionesterritorio/191019-CNPV-presentacion-Caldas-Manizales.pdf

DNP. (2012). Política Nacional de Espacio Público. Departamento Nacional de Planeación. Consejo Nacional de Política Económica y Social. CONPES 3718. Recuperado el 22 de octubre de 2018 de https://www.dadep.gov.co/sites/default/files/marco-legal/1_politica_nacional_ep_1.pdf.

Dobbs, C., Hernández-Moreno, Á., Reyes-Paecke, S., \& Miranda, M. D. (2018). Exploring temporal dynamics of urban ecosystem services in Latin America: The case of Bogota (Colombia) and Santiago (Chile). Ecological Indicators, 85, 10681080. https://doi.org/10.1016/j.ecolind.2017.11.062

French, E. L., Birchall, S. J., Landman, K., \& Brown, R. D. (2019). Designing public open space to support seismic resilience: A systematic review. International Journal of Disaster Risk Reduction, 34, 1-10. https://doi.org/10.1016/j.ijdrr.2018.11.001

Gehl, J. (2014). Ciudades para la gente. Infinito.

Giraldo-Ospina, T., \& Vásquez-Varela, L. R. (2021). Distribución e indicadores de cobertura y accesibilidad del espacio público en Manizales, Colombia. Cuadernos de Geografía: Revista Colombiana de Geografía, 30(1), 158-177. 
Gómez Orea, D., \& Gómez Villarino, A. (2013). Ordenación territorial (3a ed.). Ediciones Mundi-Prensa.

Gómez, F. (2005). Las zonas verdes como factor de calidad de vida en ciudades. Ciudad y Territorio Estudios Territoriales, 37, 417-436.

Grafakos, S., Tsatsou, A., D’Acci, L., Kostaras, J., López, A., Ramirez, N., \& Summers, B. (2019). Exploring the Use of Land Value Capture Instruments for Green Resilient Infrastructure Benefits: A Framework Applied in Cali, Colombia. Lincoln Institute of Land Policy. Recuperado el 11 de junio de 2021 de https://www.lincolninst.edu/sites/default/files/pubfiles/grafakos_wp19sg1.pdf

Haughton, G. (1997). Developing sustainable urban development models. Cities, 14(4), 189-195. https://doi.org/10.1016/S0264-2751(97)00002-4

Hernández, A. (2009). Calidad de vida y medio ambiente urbano. Indicadores locales de sostenibilidad y calidad de vida urbana. Revista INVI, 24(65), 79-111.

Jansson, M., Vogel, N., Fors, H., Dempsey, N., Buijis, A. E., \& Randrup, T. B. (2020). Defining urban open space governance and management. In M. Jansson, \& T. B. Randrup (Eds.), Urban Open Space Governance and Management. Routledge, Taylor \& Francis Group. https://doi.org/10.4324/9780429056109-3

Kaw, J. K., Lee, H., \& Wahba, S. (2020). The hidden wealth of cities. Creating, Financing, and Managing Public Spaces. World Bank. Recuperado el 21 de febrero de 2021 de http://hdl.handle.net/10986/33186

Koren, D., \& Rus, K. (2019). The Potential of Open Space for Enhancing Urban Seismic Resilience: A literature Review. Sustainability, 11(21), 5942. https://doi.org/10.3390/su11215942

Lee, A. C. K., \& Maheswaran, R. (2011). The health benefits of urban green spaces: A review of the evidence. Journal of Public Health, 33(2), 212-222.

Linares, L. A. (2018). The paradoxes of informalizing street trade in the Latin American city. International Journal of Sociology and Social Policy, 38(7-8), 651-672. https://doi.org/10.1108/IJSSP-09-2017-0119

López, L., Sepúlveda, W., \& Gómez, J. (2018). Percepción del paisaje desde la mirada del turista de algunos espacios de transformación urbana de Medellín, Colombia. Territorios, 0(39), 175-201.

https://doi.org/10.12804/revistas.urosario.edu.co/territorios/a.5629

Macedo, J., \& Haddad, M. A. (2016). Equitable distribution of open space: Using spatial analysis to evaluate urban parks in Curitiba, Brazil. Environment and Planning B: Planning and Design, 43(6), 1096-1117.

Madanipour, A., Knierbein, S., \& Degros, A. (2014). Políticas para el espacio público en las ciudades europeas. 23.

Malone, M. F. T., \& Dammert, L. (2020). The police and the public: Policing practices and public trust in Latin America. Policing and Society. https://doi.org/10.1080/10439463.2020.1744600

Manizales cómo vamos (2019). Informe de calidad de vida Manizales. Recuperado el 8 de agosto de 2021 de https://manizalescomovamos.org/documentos/

Manizales. (2001, 4 de octubre). Acuerdo Municipal 508 de 2001. Por medio del cual se adopta el Plan de Ordenamiento Territorial del municipio de Manizales.

Manizales. (2003, 24 de diciembre). Acuerdo Municipal 573 de 2003. Por medio del cual se modifica el Plan de Ordenamiento Territorial del municipio de Manizales, acuerdo 508 de octubre 12 de 2001.

Manizales. (2007, septiembre). Acuerdo Municipal 663 de 2007. Por medio del cual se hace una revisión excepcional al Plan de Ordenamiento Territorial del municipio de Manizales.

Manizales. (2017, 2 de agosto). Acuerdo Municipal 958 de 2017. Por el cual se adopta la revisión ordinaria de largo plazo del Plan de Ordenamiento Territorial del municipio de Manizales.

Mayorga, J. M. (2018). Medición de la cobertura y la accesibilidad del espacio público en Bogotá, Medellín y Cali. Cuadernos de Vivienda y Urbanismo, 11(22), 1-14.

Medellín cómo vamos (2016). Así es Medellín_Medellín. Cómo Vamos. Recuperado el 10 de abril de 2020 de https://www.medellincomovamos.org/la-ciudad/

Medellín cómo vamos. (2019). Informe de calidad de vida de Medellín, 2018. Movilidad y espacio público (p. 22). Pregón SAS. 
Medellín. (1999, 23 de diciembre). Acuerdo 62 de 1999. Por el cual se adopta el Plan de Ordenamiento Territorial del municipio de Medellín.

Medellín. (2006, agosto). Acuerdo 46 de 2006. Por el cual se revisa y ajusta el Plan de Ordenamiento Territorial para el Municipio de Medellín y se dictan otras disposiciones.

Medellín. (2014, 17 de diciembre). Acuerdo 48 de 2014. Por medio del cual se adopta la revisión y ajuste de largo plazo del Plan de Ordenamiento Territorial del Municipio de Medellín y se dictan otras disposiciones complementarias.

Mesa Carranza, J. A., \& López Bernal, O. (2016). Propuesta de un sistema de indicadores para evaluar la calidad visual del paisaje urbano en asentamientos informales. Revista de Arquitectura, 18, 35-47.

Mora Eusse, J. S. (2019). Más espacio público efectivo por habitante en Medellín. En Medellín cuenta. Medellín cuenta. Recuperado el 25 de agosto de 2020 de https://www.medellincuenta.com/?NavigationTarget=navurl://ac2d4a7592bbd5762dcd7f81d5331104

Ojeda Casares, S., de Oliveira Neves, G., Schröder, C., \& Hurtado Rodríguez, C. (2008). Equidad territorial en Andalucía (Número January). Instituto de Estadística de Andalucía.

ONU, Organización de las Naciones Unidas. (2016). Nueva Agenda Urbana. Secretaría de Hábitat III. Recuperado el 30 de enero de 2018 de http://habitat3.org/wp-content/uploads/NUA-Spanish.pdf

Páramo, P., \& Burbano, A. M. (2012). Valoración de las condiciones que hacen habitable el espacio público en Colombia. Territorios, 28, 187-206.

Pérez Medina, S. (2018). Legislación urbana y oferta de áreas verdes de recreación en Mérida, Yucatán. Estudios Demográficos y Urbanos, 33(3), 671-698. https://doi.org/10.24201/edu.v33i3.1813

Parques Nacionales Naturales de Colombia - PNNC. (2015). Áreas protegidas territorios para la vida y la paz. Tomo II Áreas protegidas, paisajes rurales y urbanos: Uniendo esfuerzos de conservación.

Pojani, D., \& Maci, G. (2015). The Detriments and Benefits of the Fall of Planning: The Evolution of Public Space in a Balkan Post-socialist Capital. Journal of Urban Design, 20(2), 251-272.

Posada, A. (2014). De la planeación a la gestión ambiental colectiva: Posibilidades en Bogotá-Colombia. Revista U.D.C.A Actualidad \& Divulgación Científica, 17(1), 257-264.

PRC. (1998). Decreto 1504 de 1998 Nivel Nacional. Presidencia de la República de Colombia. Diario Oficial No. 43357 de agosto 6 de 1998. Presidencia de la República de Colombia. Recuperado el 14 de febrero de 2020 de https://www.alcaldiabogota.gov.co/sisjur/normas/Norma1.jsp?i=1259

Rigolon, A., Browning, M., Lee, K., \& Shin, S. (2018). Access to Urban Green Space in Cities of the Global South: A Systematic Literature Review. Urban Science, 2(67), 67-91.

Sarmiento, O. L., Díaz del Castillo, A., Triana, C. A., Acevedo, M. J., Gonzalez, S. A., \& Pratt, M. (2017). Reclaiming the streets for people: Insights from Ciclovías Recreativas in Latin America. Preventive Medicine, 103, S34-S40.

https://doi.org/10.1016/j.ypmed.2016.07.028

Smolka, M. (2013). Implementación de la recuperación de plusvalías en América Latina.

Talavera-Dávila, H. V. (2017). Plataforma de cohesión espacial para la inclusión social. Bitácora Urbano Territorial, 27(2), 79-89. https://doi.org/10.15446/bitacora.v27n2.47577

Thompson, C. W. (2002). Urban open space in the 21st century. Landscape and Urban Planning, 60(2), 59-72. https://doi.org/10.1016/S0169-2046(02)00059-2

Zuindeau, B. (2007). Territorial equity and sustainable development. Environmental Values, 16(2), 253-268. https://doi.org/10.3197/096327107780474564

Editor: Luís Alberto Salinas Arreortua

Recibido: 28 abr. 2021

Aprobado: 29 jul 2021 